\title{
PROGRESSIVE BITSTREAM TRANSMISSION OVER TANDEM CHANNELS
}

\author{
Farzad Etemadi, Homayoun Yousefi'zadeh, Hamid Jafarkhani \\ Department of EECS \\ University of California, Irvine \\ [fetemadi, hyousefi, hamidj] duci.edu
}

\begin{abstract}
We propose a novel distortion minimization technique for the transmission of a packetized progressive bitstream. We consider tandem channels introducing bit errors and packet erasures. We formalize the distortion minimization problem as a constrained optimization problem and propose an algorithm that optimally allocates the available budget between the bit error and packet loss protection components. We show that our proposed optimization technique is robust and has a linear complexity in the transmission rate. Numerical results show the effectiveness of the proposed algorithm.
\end{abstract}

\section{INTRODUCTION}

Embedded coding is the ability of a source coder to allow a decoder to progressively reconstruct its data at different bit rates from the prefixes of a single bitstream. Practical implementations of progressive encoders include SPIHT [7] for still image coding, and scalable H.264/AVC [8] for video coding. The embedded capability of progressive encoders, however, comes at the expense of high sensitivity to transmission noise and the possibility of error propagation. Therefore, progressive transmission over noisy channels has to be accompanied with appropriate channel coding or joint source-channel coding schemes.

Joint source-channel coding of progressive bitstreams has been studied by many researchers. Concatenated coding $[2,9]$, dynamic programming [2, 3, 4], exhaustive search [9], and gradient-based optimization [1, 6, 13] are among the techniques used to solve different variants of the latter problem. In [13], we applied a gradient-based optimization method to the transmission of progressive bitstreams over tandem channels characterized by bit errors and packet erasures.

The above-mentioned algorithms are either sub-optimal in the distortion sense, or have a best case $\mathcal{O}(N \log N)$ complexity in the number of packets $N$. Gradient-based techniques are also sensitive to the initial conditions, and may fail to find the global optimum. In [5], we introduced a linear complexity distortion minimization technique for chan- nels that only introduce bit errors. This latter algorithm is globally optimal for sources with exponential DistortionRate (D-R) function (e.g., Gauss-Markov), and is also very effective for arbitrary progressively encoded sources. Moreover, the proposed algorithm eliminates the need for a closedform and smooth D-R function of the source that is used in many of the gradient-based techniques such as our work of [13].

The low complexity of our proposed algorithm in [5] makes it particularly useful in applications involving realtime deadlines such as video transmission. Our objective in this paper is to extend the latter algorithm to the case of tandem channels, where both bit errors and packet erasures are present. We propose a linear-complexity algorithm that minimizes the distortion for a tandem channel. Moreover, we show that our proposed algorithm can optimally allocate the available budget between the bit error and packet erasure components. We thus eliminate the need for a design parameter to specify the latter budget allocation which was a shortcoming of [13].

The structure of this paper is as follows. In Section 2, we formulate our distortion-optimal problems and present our proposed solutions to them. We present our numerical results in Section 3. Finally, Section 4 concludes this paper.

\section{ANALYSIS}

We first consider the problem of transmitting a progressively encoded bitstream over a channel that only introduces bit errors. We use $N$ packets of length $L$ symbols with a total budget of $B_{T}=N L$ symbols. As shown in Fig. 1(a), the $i^{\text {th }}$ packet consists of $C_{i}$ channel coding symbols together with $b_{i}$ data symbols. The expected distortion of the $N$ packet transmitted bitstream can be written as [13]:

$$
J^{N}=\sum_{i=1}^{N+1} D_{i-1} \Psi_{i} \prod_{j=1}^{i-1}\left(1-\Psi_{j}\right)
$$

where $\Psi_{i}$ is the failure probability of packet $i$ and $D_{i}$ is the distortion associated with the first $i$ packets. The boundary 

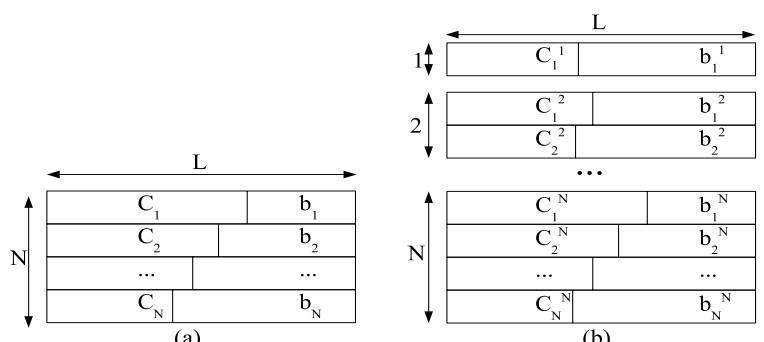

(b)

Fig. 1. Optimization problem with bit errors only (a) original optimization problem (b) packet by packet optimization problem.

conditions for the above equations are $\Psi_{N+1}=1$ and $D_{0}=$ $\sigma^{2}$ where $\sigma^{2}$ is the source variance. Variables $D_{i}$ and $\Psi_{i}$ depend on the choice of the source coder, channel model as well as error statistics, and the choice of $C_{i}$ 's. The goal is to find a set of $C_{i}$ 's that minimize this distortion.

A global search over all possible parity combinations requires $L^{N}$ cost function evaluations which is impractical. We can reduce this number by making an unequal error protection (UEP) assumption in the form of $C_{1} \geq C_{2} \geq$ $\cdots \geq C_{N}$ which is quite reasonable for a progressively encoded bitstream. Even with the UEP assumption, the number of function evaluations increases very quickly with $L$ and $N$. As an alternative approach, one can try to solve a constrained nonlinear optimization problem in the form of:

$$
\begin{aligned}
\underset{C_{1}, \cdots, C_{N}}{\min } & J^{N} \\
\text { Subject To: } & 0 \leq C_{i}<L, \quad i \in\{1, \cdots, N\}
\end{aligned}
$$

In [5], we solved the optimization problem (2) using a packet by packet approach as shown in Fig. 1(b). We solve $N$ single-variable optimization problems instead of solving an optimization problem with $N$ decision variables. The $i^{\text {th }}$ optimization problem consists of $i$ packets, but only the first packet has to be optimized. Let us use superscript $i$ as an index for the $i^{\text {th }}$ optimization problem. $C_{j}^{i}$ and $\Psi_{j}^{i}$ then represent the number of channel coding symbols and the failure probability of the $j^{t h}$ packet in the $i^{\text {th }}$ optimization problem, respectively. Our optimization algorithm can be formalized as the following:

\section{Algorithm $\mathrm{A}_{1}$}

- Find $C_{1}^{1} \in\{0,1, \cdots, L-1\}$ that minimizes the expected distortion for a single packet.

- For $i=2, \cdots, N$

- Set $C_{j}^{i}=C_{j-1}^{i-1} \quad j=2, \cdots, i$.

- Find $C_{1}^{i} \in\{0, \cdots, L-1\}$ that minimizes the expected distortion for $i$ packets.
- $\left\{C_{1}^{N}, \cdots, C_{N}^{N}\right\}$ is the optimal solution.

It can be proved that $\mathbf{A}_{\mathbf{1}}$ is optimal for the class of sources satisfying the following condition [5]:

$$
D(n+\Delta n)=\frac{D(n) D(\Delta n)}{D(0)} \quad \Delta n \leq L
$$

In the above equation, $D(n)$ is the distortion associated with the first $n$ information symbols. As an example, a GaussMarkov source has an asymptotic D-R function in the form of an exponential $D(n)=\alpha e^{-\beta n}$ that satisfies the optimality condition (4). Simulation results show that $\mathbf{A}_{\mathbf{1}}$ also provides good results for sources characterized by piecewise exponential D-R functions. The latter condition is practically met for a wide range of sources and as a result, $\mathbf{A}_{\mathbf{1}}$ can be applied to all such sources.

The worst-case complexity of $\mathbf{A}_{\mathbf{1}}$ is $\mathcal{O}(N L)$. In other words, it is linear in the transmission rate. Notice that by invoking the UEP assumption, we can limit the search space for $C_{1}^{i}$ to the set $\left\{C_{2}^{i}, \cdots, L-1\right\}$. This will usually not compromise the optimality of the algorithm. The search space can be further limited based on the allowable code rates.

In deriving $\mathbf{A}_{1}$, we have made no assumptions on the channel model and the channel coding scheme used. $\mathbf{A}_{\mathbf{1}}$ is applicable to any system so long as the bitstream is progressively encoded. While the choice of the source coder, channel coder, and the transmission medium is reflected in the variables $D_{i}$ and $\Psi_{i}$, it does not change the algorithm. Packet failure rates, $\Psi_{i}$, can be either calculated exactly as in [13] or can be obtained through numerical simulations [6].

The by-product of the recursive optimal solution for $N$ packets is the optimal solution for $k$ packets where $1 \leq k \leq$ $N-1$. This property is useful in applications involving different levels of Quality of Service (QoS) associated with different transmission rates. Performing the optimization for the maximum transmission rate automatically provides the optimal channel coding rates for all of the intermediate transmission rates.

We notice that the convergence of the search algorithm is guaranteed for all values of $N$ and $L$. Consequently, the convergence of $\mathbf{A}_{\mathbf{1}}$ is not affected by the choice of initial conditions unlike gradient-based algorithms.

We now assume that the channel introduces packet erasures in addition to bit errors. We consider a product code as shown in Fig. 2. The first $N$ packets contain data and their associated parity bits for bit error protection while an additional $N_{E}$ packets are used for erasure recovery. The first $N$ symbols of every column form the data portion of a maximum distance separable (MDS) code that is used for erasure recovery. The MDS property of the column code facilitates the recovery of the entire packet set as long as the number of received packets is $N$ or more [12]. We now define a new 


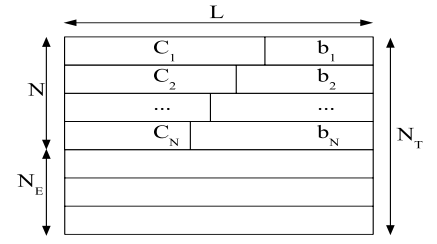

Fig. 2. Optimization problem with bit errors and packet erasures

probabilistic cost function that includes the effects of packet erasures as:

$$
J=P_{R}(N) J^{N}+\left(1-P_{R}(N)\right) D_{0}
$$

where $P_{R}(N)$ is the packet set recovery probability and is defined as the probability of receiving $N$ or more packets out of $N_{T}$ transmitted packets. $P_{R}(N)$ can be further written as $P_{R}(N)=1-\sum_{k=0}^{N-1} P\left(N_{T}, k\right)$ where $P\left(N_{T}, k\right)$ is the probability of receiving $k$ packets out of $N_{T}$ transmitted packets. We note that Equation (5) implicitly assumes that no packets have been received if the number of received packets is less than $N$. Even though in this case we can not recover the packet set, we can still decode the bitstream if the first $k$ packets have been received with $k$ being arbitrary. Therefore, Equation (5) provides a somewhat pessimistic estimate of the expected distortion. The new optimization problem is then formulated as:

$$
\begin{array}{cl}
\underset{C_{1}, \cdots, C_{N}, N}{\min } & J \\
\text { Subject To: } & 0 \leq C_{i}<L, \quad i \in\{1, \cdots, N\} \\
& 1 \leq N \leq N_{T}
\end{array}
$$

with a total available budget of $B_{T}=N_{T} L$, where $N_{T}=$ $N+N_{E}$. A brute-force solution of this optimization problem involves $N_{T}$ optimizations and is extremely inefficient unless a fast optimization technique is found for minimizing $J^{N}$. Moreover, note that the number of data packets, $N$, is itself an optimization variable. Since the optimization problem (6) has a variable number of optimization variables, it can not be solved using the traditional optimization techniques.

We now introduce an alternative solution to the constrained optimization problem of (6) relying on our solution to the bit-error only problem (2). The algorithm $\mathbf{A}_{1}$ to minimize $J^{N}$ has the desired property that it also provides the global minimum of $J^{k}$ for all $1 \leq k \leq N$. We use this property to develop a low-complexity exhaustive search algorithm for minimizing the cost function of (6). We formalize our algorithm as the following:

\section{Algorithm $\mathrm{A}_{2}$}

- For a given $L$ and channel characteristics, calculate $P_{R}(N)$ for every $N \in\left\{1,2, \cdots, N_{T}\right\}$.
- Minimize $J^{N_{T}}$ using $\mathbf{A}_{\mathbf{1}}$. Save all of the intermediate optimal cost functions $J^{N}$ for $1 \leq N \leq N_{T}$ as well as the corresponding optimal solutions for $C_{i}$ 's.

- Using Equation (5), evaluate $J$ for $1 \leq N \leq N_{T}$ and find its minimum. The corresponding $N$ and $C_{i}$ 's provide the solution to (6).

Notice that the first step of $\mathbf{A}_{\mathbf{2}}$ does not depend on the bitstream and can be performed off-line for a given channel. $\mathbf{A}_{2}$ requires only one optimization with a complexity of $\mathcal{O}\left(N_{T} L\right)$. In other words, it has a linear complexity in the transmission rate. We note that if we use the sub-optimal equal protection scheme for the second step of $\mathbf{A}_{\mathbf{2}}$, the complexity is still $\mathcal{O}\left(N_{T} L\right)$ since in that case, we need to perform a two-dimensional search over $L$ possible code rates and $N_{T}$ possible budget allocations. Finally, we notice that $\mathbf{A}_{2}$ is a globally optimal algorithm since it performs an efficient exhaustive search over all possible budget allocations.

\section{NUMERICAL RESULTS}

In this section, we present the simulation results of our proposed algorithm. We use the $512 \times 512$ gray scale Barbara image encoded progressively using the SPIHT encoder, [7]. The operational D-R curve that is used in the optimization algorithm is implemented as the following set of $(R, D)$ pairs: $\{(0,2227.8),(0.03,365.9),(0.35,74.7),(0.76,24.4)$, $(2.26,2.8),(3,1.6)\}$ where the rate $R$ is expressed in $b p p$. The D-R curve is approximated as a piecewise exponential function between the set of above points. This justifies the use of the algorithm for practical implementations where an accurate estimation of the D-R curve may not be available. In practice, this lookup table can be generated during the encoding process based on the wavelet coefficient quantization errors [7]. The time-varying bit error rate of a fading channel is modeled using the two-state Gilbert-Elliott channel. The transition probabilities for the GOOD to BAD and BAD to GOOD state transitions are 0.00127 and 0.125 , respectively. Per state symbol error rates are derived from the per state signal-to-noise ratios. We use the relationship $S N R_{G}=10 S N R_{B}$ in order to distinguish between the signal-to-noise ratios of the GOOD and BAD states. Channel coding is done using rate compatible punctured ReedSolomon codes over $G F(256)$ with a symbol size of one byte. We refer the reader to [13] for a detailed description of the channel model and the calculation of the loss probabilities $\Psi_{i}$. A packet length of $L=200$ bytes is used in our experiments.

Fig. 3 shows the performance of algorithm $\mathbf{A}_{1}$ for a channel that introduces bit errors only. The SPIHT source coder performance is also shown as a point of reference. A tandem channel is considered in our next experiment. Packet erasures are modeled using a Gilbert channel. The 


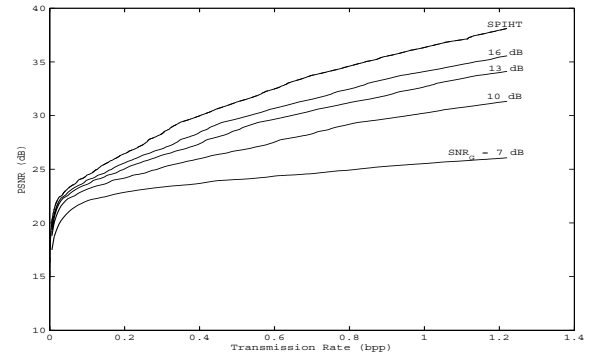

Fig. 3. Numerical results for the transmission of the Barbara image over a channel with bit errors only
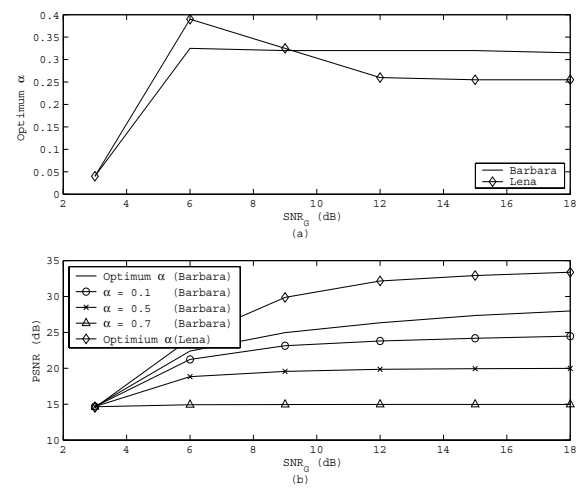

Fig. 4. Tandem channel with a transmission rate of $1.22 \mathrm{bpp}$ (a) Optimum $\alpha$ (b) Expected PSNR

Gilbert channel is essentially the same as the Gilbert-Elliot channel with constant values of GOOD and BAD state error rates of $\varepsilon_{G}=0$ and $\varepsilon_{B}=1$, respectively. We have used a total of $N_{T}=200$ packets which translates into a transmission rate of $1.22 \mathrm{bpp}$. The GOOD to BAD and BAD to GOOD transition probabilities of the erasure channel are 0.1 and 0.125 , respectively. The parameter $\alpha=\frac{N}{N_{T}}$ defines the fraction of the budget allocated for bit error protection and data transmission, while $1-\alpha$ represents the fractional budget for erasure protection. The results of algorithm $\mathbf{A}_{\mathbf{2}}$ for the optimal budget allocation are shown in Fig. 4 (a). We note that for moderately high values of $S N R_{G}$, the optimal value of $\alpha$ is relatively constant. In Fig. 4 (b), we have compared the expected PSNR for the optimal $\alpha$ with a number of constant budget allocation schemes. These results show that our proposed algorithm is indeed optimal when both bit errors and packet erasures are present. Fig. 4 also shows similar results for our experiments using the Lena image.

\section{CONCLUSION}

In this paper, we proposed a novel distortion minimization technique for the transmission of packetized progressive bit- streams. We considered tandem channels subject to bit errors and packet erasures. We formulated the distortion minimization problem as a constrained optimization problem and showed that our proposed algorithm has a linear complexity in the transmission rate and can optimally allocate the available budget between the bit error and packet erasure components. We numerically showed the effectiveness of our proposed algorithm for arbitrary progressively encoded sources.

\section{REFERENCES}

[1] A. Appadwedula, D.L. Jones, K. Ramchandran, I. Konzintsev, "Joint Source Channel Matching for A Wireless Communications Link,” In Proc. IEEE ICC, 1998.

[2] B.A. Banister, B. Belzer, T.R. Fischer, "Robust Image Transmission Using JPEG2000 and Turbo-Codes," IEEE Sig. Proc. Letters, April 2002.

[3] V. Chande, H. Jafarkhani, N. Farvardin, "Image Communication over Noisy Channels with Feedback," In Proc. IEEE ICIP, 1999.

[4] V. Chande, N. Farvardin, "Progressive Transmission of Images over Memoryless Channels," IEEE JSAC, June 2000.

[5] F. Etemadi, H. Yousefi'zadeh, H. Jafarkhani, "A LinearComplexity Distortion Optimal Scheme for Transmission of Progressive Packetized Bitstreams," IEEE Sig. Proc. Letters, May 2005.

[6] A. Nosratinia, J. Lu, B. Aazhang, "Source-Channel Rate Allocation for Prgogressive Transmission of Images," IEEE Trans. Comm., Feb. 2003.

[7] A. Said, W.A. Pearlman, "A New Fast and Efficient Image Codec Based on Set Partitioning in Hierarchical Trees," IEEE Trans. CSVT, June 1996.

[8] H. Schwarz, D. Marpe, T. Wiegand, "Scalable Extension of H.264/AVC," ISO/IEC JTC1/SC29/WG11 JVT-K023, March 2004.

[9] P.G. Sherwood, K. Zeger, "Progressive Image Coding for Noisy Channels,” IEEE Sig. Proc. Letters, July 1997.

[10] V. Stankovic, R. Hamzaoui, Y. Charfi, Z. Xiong, "Real-Time Unequal Error Protection Algorithms for Progressive Image Transmission,” IEEE JSAC, Dec. 2003.

[11] V. Stankovic, R. Hamzaoui, D. Saupe, "Fast Algorithm for Rate-Based Optimal Error Protection of Embedded Codes," IEEE Trans. Comm., Nov. 2003.

[12] S. Wicker, "Error Control Systems for Digital Communications and Storage," Prentice-Hall, 1995.

[13] H. Yousefi'zadeh, H. Jafarkhani, F. Etemadi, "DistortionOptimal Transmission of Progressive Images over Channels with Random Bit Errors and Packet Erasures," In Proc. IEEE DCC, 2004. 